\title{
A general relation between sums of cubes and triangular pyramidal numbers
}

\begin{abstract}
Let $\mathrm{ck}(\mathrm{m})$ denote the number of representations of integer $\mathrm{m}$ as a sum of $\mathrm{k}$ cubes and $\mathrm{pk}(\mathrm{m})$ denote the number of representations of integer $\mathrm{m}$ as a sum of $\mathrm{k}$ triangular pyramidal numbers. We give a relation $\mathrm{pk}(\mathrm{m})=\operatorname{coddk}(3)$ where $3=48 \mathrm{~m} \ddot{\mathrm{i}} 24 \mathrm{n}+2 \tilde{\mathrm{n}}+\mathrm{k}$ and coddk (3) denotes the number of representations of integer 3 as a sum of $\mathrm{k}$ odd cubes, for a single value of $\mathrm{m}$. A general relation between number of representations between $\mathbb{k k i}=1$ xsi and its associated polytopic numbers for any orders of $\mathrm{s}$, is also given.
\end{abstract}

Keyword: Number of representations; Sum of cubes; Sum of triangular pyramidal numbers 